\title{
Ureteroscopy-assisted Percutaneous Kidney Access Made Easy: First Clinical Experience with a Novel Navigation System Using Electromagnetic Guidance (IDEAL Stage 1)
}

\author{
Estevao Lima $^{a, *}$, Pedro L. Rodrigues ${ }^{a, b}$, Paulo Mota $^{a}$, Nuno Carvalho ${ }^{a}$, Emanuel Dias $^{a}$, \\ Jorge Correia-Pinto ${ }^{a}$, Riccardo Autorino ${ }^{a}$, João L. Vilaça ${ }^{a, b}$ \\ ${ }^{a}$ Department of CUF Urology, Surgical Sciences Research Domain, Life and Health Sciences Research Institute, School of Medicine, University of Minho, Braga, \\ Portugal; ' DIGARC, Polytechnic Institute of Cávado and Ave, Barcelos, Portugal
}

\section{Article info}

\section{Article history:}

Accepted March 7, 2017

Associate Editor:

Alexandre Mottrie

\section{Keywords:}

Combined access

Kidney puncture

Surgical navigation

Kidney access

Percutaneous nephrolithotomy

Image-guided surgery

Real time

\section{Please visit}

www.europeanurology.com and www.urosource.com to view the accompanying video.

\begin{abstract}
Background: Puncture of the renal collecting system represents a challenging step in percutaneous nephrolithotomy (PCNL). Limitations related to the use of standard fluoroscopic-based and ultrasound-based maneuvers have been recognized.

Objectives: To describe the technique and early clinical outcomes of a novel navigation system for percutaneous kidney access.

Design, setting, and participants: This was a proof-of-concept study (IDEAL phase 1) conducted at a single academic center. Ten PCNL procedures were performed for patients with kidney stones.

Surgical procedure: Flexible ureterorenoscopy was performed to determine the optimal renal calyx for access. An electromagnetic sensor was inserted through the working channel. Then the selected calyx was punctured with a needle with a sensor on the tip guided by real-time three-dimensional images observed on the monitor.

Outcome measurements and statistical analysis: The primary endpoints were the accuracy and clinical applicability of the system in clinical use. Secondary endpoints were the time to successful puncture, the number of attempts for successful puncture, and complications.

Results and limitations: Ten patients were enrolled in the study. The median age was $47.1 \mathrm{yr}(30-63)$, median body mass index was $22.85 \mathrm{~kg} / \mathrm{m}^{2}$ (19-28.3), and median stone size was $2.13 \mathrm{~cm}(1.5-2.5 \mathrm{~cm})$. All stones were in the renal pelvis. The Guy's stone score was 1 in nine cases and 2 in one case. All 10 punctures of the collecting system were successfully completed at the first attempt without X-ray exposure. The median time to successful puncture starting from insertion of the needle was $20 \mathrm{~s}$ (range 15-35). No complications occurred.

Conclusions: We describe the first clinical application of a novel navigation system using real-time electromagnetic sensors for percutaneous kidney access. This new technology overcomes the intrinsic limitations of traditional methods of kidney access, allowing safe, precise, fast, and effective puncture of the renal collecting system. Patient summary: We describe a new technology allowing safe and easy puncture of the kidney without radiation exposure. This could significantly facilitate one of the most challenging steps in percutaneous removal of kidney stones.
\end{abstract}

(C) 2017 European Association of Urology. Published by Elsevier B.V. All rights reserved.

\footnotetext{
* Corresponding author. Department of CUF Urology, Surgical Sciences Research Domain, Life and Health Sciences Research Institute, School of Medicine, University of Minho, 4709-057 Braga, Portugal. Tel. +351 919327639; Fax: +351253604809.

E-mail address: estevaolima@med.uminho.pt (E. Lima).
} 


\section{Introduction}

First described in 1976, percutaneous nephrolithotomy (PCNL) has gained an established role in the contemporary surgical management of urolithiasis [1,2]. Both European Urology Association and American Urological Association guidelines recommend PCNL as the treatment option for larger renal calculi $[3,4]$.

The PCNL procedure includes several steps: percutaneous puncture of a renal calyx, tract dilatation, nephroscopy, and stone fragmentation and removal [5]. Among these steps, obtaining safe and appropriate access to the kidney represents one of the most difficult, and can ultimately impact the outcomes of the procedure [6]. Fluoroscopy and ultrasound, alone or combined, are the methods most often used to guide puncture of the renal collecting system [7]. However, access techniques based on these methods remain suboptimal [8]. Moreover, concerns related to radiation exposure when fluoroscopy is used have been raised [9].

We recently described experimental use of a novel visual-assisted navigation system in a porcine model using real-time electromagnetic sensors to allow kidney puncture for PCNL [10]. Here we report the first use of this device in humans and describe in detail the surgical technique and analyze early clinical outcomes.

\section{Patients and methods}

\subsection{Study design}

This was a prospective proof-of-concept phase 1 study according to the IDEAL criteria [11]. All procedures were performed by a single staff surgeon (E.L.) at the CUF Department of Urology of Braga Hospital (Braga, Portugal), which is a tertiary academic medical center. All patients gave their written informed consent to test our navigation system for renal colleting system puncture after the risks, benefits, and alternatives were discussed. Institutional review board approval was obtained before the start of the study. Patients were specifically informed that this was first clinical application of this novel system. The primary endpoint was the clinical applicability of the system for PCNL. Secondary endpoints were assessment of accuracy (in terms of time to successful puncture and number of attempts for successful puncture) and safety (in terms of puncture-related complications).

\subsection{Patient selection criteria}

Inclusion criteria for patient selection were: age older than $18 \mathrm{yr}$; stone in the renal pelvis; stone size $\leq 2.5 \mathrm{~cm}$; and Guy's stone score of 1-2 [12].

Exclusion criteria were: obese patient (body mass index [BMI] $>30 \mathrm{~kg} / \mathrm{m}^{2}$ ); lower calyx fully engaged with stones; bilateral stones, solitary kidney, renal insufficiency, anatomic renal anomalies, stone size $>2.5 \mathrm{~cm}$, and Guy's stone score of 3-4 [12].

\subsection{Perioperative management}

Patients underwent standard preoperative anesthesia testing. A computed tomography (CT) urogram with three-dimensional (3D) reconstruction was also obtained. Patients with negative urine cultures were treated with a single prophylactic dose of a broad-spectrum antibiotic.

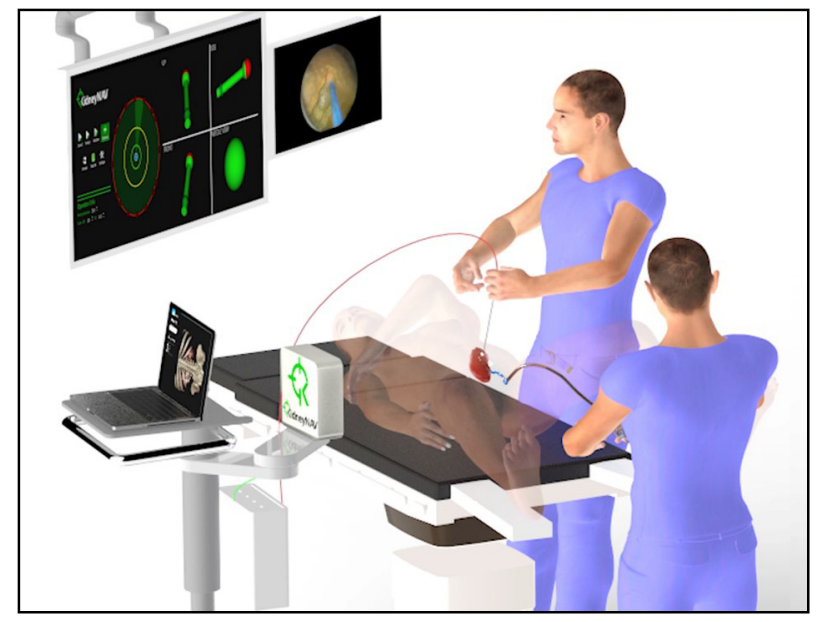

Fig. 1 - Surgical set-up for puncture of the renal collecting system using electromagnetic sensors. A research group from the University of Minho (Braga, Portugal) has patented this new navigation system. The technology consists of the following components: (1) software for surgical guidance developed specifically for this work, which acts as a control station by gathering and processing information from different equipment needed for puncture for percutaneous nephrolithotomy; (2) an electromagnetic field generator placed on the opposite side of the puncture, close to the patient; (3) one $18 \mathrm{G}$ needle and one ureteral catheter, both with an electromagnetic sensor on the tip; (4) a monitor with a four-view 3D representation of the trajectory orientation and position of the needle and catheter; and (5) a monitor displaying the ureterorenoscopy video image.

\subsection{Instrumentation}

The commercially available Aurora EMT system (Northern Digital, Waterloo, Canada) was used to track the catheter and needle tip inside the ureteral and kidney calyx. This navigation system comprises the following components (Fig. 1):

(1) A planar, low-intensity, and varying electromagnetic field generator that establishes a tracking volume.

(2) Two sensor interface units (SIUs) that act as analog-to-digital converters and amplifiers of the electrical signals from the sensors to a system control unit (SCU). The SIUs decrease the possibility of electromagnetic interference in the operating room. The SCU transmits spatial data to a computer for subsequent processing and navigation using the software described below.

(3) One Chiba needle $(18 \mathrm{G} / 180 \mathrm{~mm})$ and one ureteral catheter of $1.1 \mathrm{~mm}$ in diameter and $2 \mathrm{~m}$ in length. Both include an Aurora EMT sensor with five degrees of freedom at its tip.

(4) 3DPuncture software (EMT kidney and ureter percutaneous access software) for surgical guidance that was developed specifically for this work using $\mathrm{C}++$ and VTK (The Visualization ToolKit). The software gathers and processes information from different equipment needed for PCNL puncture: images from the videoureterorenoscope, and the orientation and position of the needle and catheter EMT sensors. It allows the surgeon to choose the correct needle orientation in real time.

\subsection{Technique}

The accompanying video illustrates the technology and provides a stepby-step description of the procedure. Under general anesthesia, the patient is placed in the supine position to allow a combined approach with flexible ureterorenoscopy and percutaneous nephroscopy. The 
generator is placed near the patient (outside the surgical field) to create the electromagnetic field for tracking the position of the catheter and needle sensors. Then a cystoscope (model 27035BA, Karl Storz, Tuttlingen, Germany) is inserted for identification of the ureteral meatus, and a hydrophilic guidewire (model AE0N35, Terumo, Shibuya, Tokyo) is inserted through it. A digital videoureterorenoscope (Karl Storz model $11278 \mathrm{VSK}$ ) is inserted, guided by the hydrophilic guidewire, up to the renal pyelocaliceal system. No ureteral access sheath is used. After removing the guidewire, a ureteral catheter with electromagnetic sensor is inserted through the working channel of the digital videoureterorenoscope. Under ureteroscopy visualization, the surgeon selects the ideal calyx for percutaneous access, and places the ureteral catheter with the electromagnetic sensor in the fornix of the calyx.

An ultrasound scan is used to verify that the renal puncture track is not obstructed by any unintended anatomical structure. The selected calyx is punctured using an $18 \mathrm{G}$ needle with an electromagnetic sensor on the tip of the stylet. The access is guided in real time by images observed on the monitor (Fig. 2). Once the needle tip is inserted into the desired calyx the proper calyceal access is confirmed ureteroscopically, and minor adjustments are made under endoscopic visualization.

To obtain the working tract for PCNL, the inner stylet containing the electromagnetic sensor is removed and a guidewire is inserted, followed by balloon dilatation and sheath placement under direct ureteroscopy visualization. The entire process is performed without fluoroscopy, and is only monitored using the digital videoureterorenoscope image.

Finally, the entire PCNL procedure is performed under ureteroscopy visualization, as described by others [13].

\section{Results}

\subsection{Study population}

Ten patients were enrolled in the study. The median age was $47.1 \mathrm{yr}$ (30-63), median BMI was $22.85 \mathrm{~kg} / \mathrm{m}^{2}$ (19-28.3), and median stone size was $2.13 \mathrm{~cm}(1.5-2.5 \mathrm{~cm})$. All stones were in the renal pelvis. The Guy's stone score was 1 in nine cases and 2 in one case.

\subsection{Outcomes}

The ten punctures of the collecting system were successfully completed. The median time to successful puncture

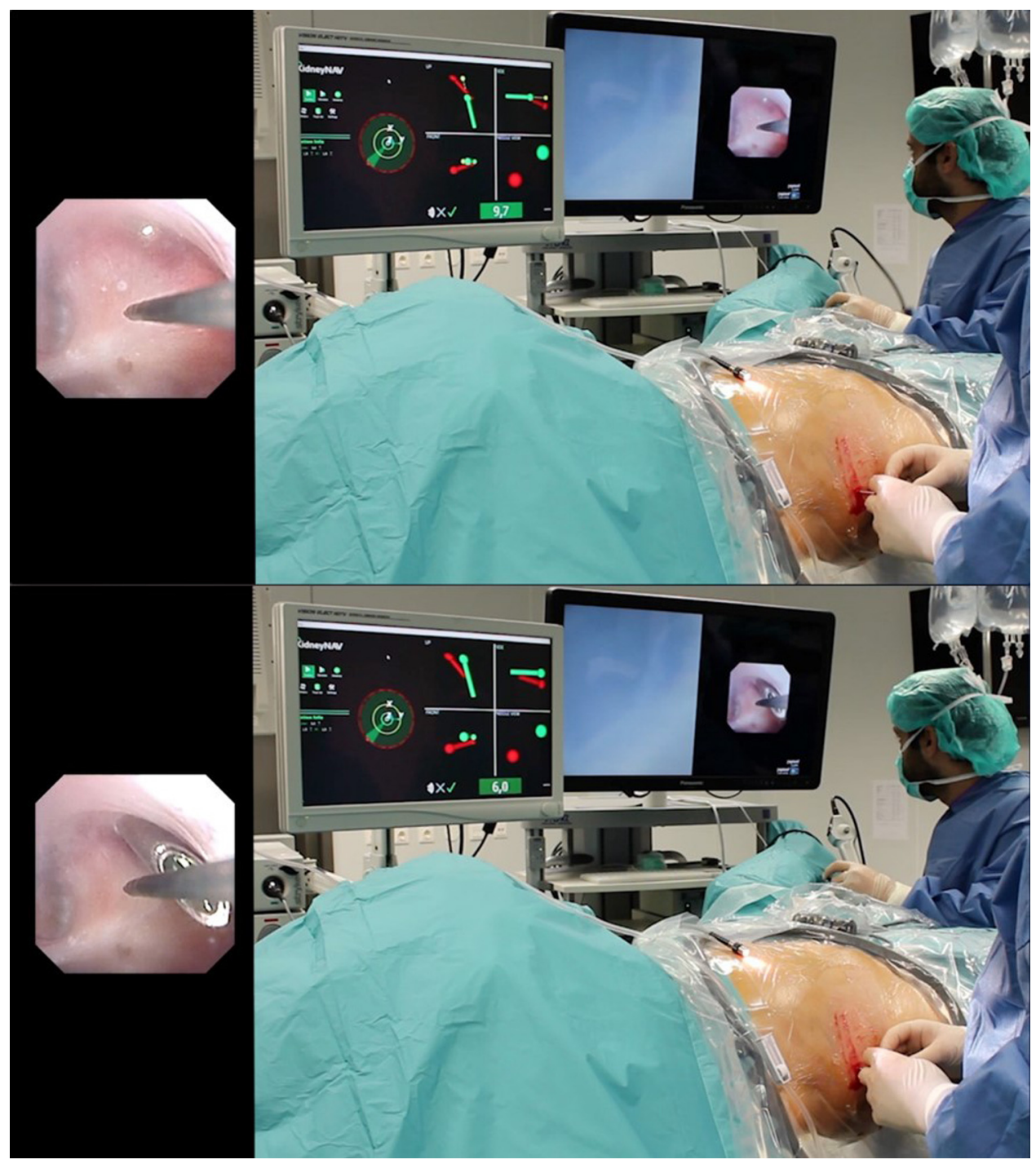

Fig. 2 - Guided by the three-dimensional navigation software on the monitor and confirmed simultaneously by ureterorenoscope images, the surgeon performs puncture of the lower calyx during human percutaneous nephrolithotomy. 
starting from insertion of the needle was 20 s (range 15-35). All punctures were successful at the first attempt. All the procedures were performed without X-ray exposure. No complications occurred.

\section{Discussion}

We report the first clinical use of a novel technique for percutaneous kidney access based on a novel tracking system using visual-assisted navigation and real-time electromagnetic sensors. We successfully demonstrated the accuracy and safety of this technology. Precise puncture of the collecting system was obtained in $100 \%$ of cases, with a median time to successful puncture of 20 s, without X-ray exposure and without any complications.

This system was previously tested in an ex vivo model and in an in vivo animal model [10]. In the preliminary investigation, six female pigs were subjected to ureteral and kidney punctures, and four punctures were performed by two surgeons in each animal, including one in the kidney and one in the middle ureter, on both sides. All 24 punctures (12 in the middle ureter and 12 in the renal calyces) were successfully carried out. The average time for puncture was $19 \mathrm{~s}$ in the kidney and $51 \mathrm{~s}$ in the ureter $(p=0.003)$. A shorter puncture planning time was recorded for expert surgeons compared those in training $(p=0.03)$.

In this procedure, we used a combined retrograde and percutaneous endoscopic approach for intrarenal surgery, which has become increasingly popular over the past few years [13]. Ureteroscopy-guided percutaneous fluoroscopic access was already suggested $20 \mathrm{yr}$ ago [14]. More recently, Alsyouf et al [15] described a technique combining ultrasound guidance with direct endoscopic visualization; they chose the ideal calyx for puncture using ureteroscopy visualization, and ultrasound served as a guide for insertion of the needle.

Using our navigation system with real-time electromagnetic sensors, the virtual track is visualized in 3D on the monitor so that the surgeon can confirm that the catheter and needle are aligned in parallel. When necessary, the surgeon can redefine the orientation of the catheter and a new virtual trajectory is then calculated. The procedure provides real-time positioning, allowing the surgeon to achieve constant perfect orientation of the needle even in the presence of anatomical deformities. Confirmation of the absence of anatomical structures along the puncture path is checked using ultrasound. This technique has the following advantages: a lack of exposure to ionizing radiation; realtime 3D images of the needle trajectory; correct needle placement and orientation in real time; greater ease of technical learning; a short execution time; the possibility of redefining the trajectory; constant monitoring via the electromagnetic sensors and the endoscopic view, allowing the surgeon to make minor adjustments; and real-time monitoring of anatomical changes. Moreover, the entire procedure can be performed in the supine position, eliminating the need for patient repositioning and thus reducing the operation time. However, we also recognize some disadvantages associated with this novel technique: a lack of visualization of surrounding anatomical structures; and potentially difficult placement of the ureteral catheter with an electromagnetic sensor in the desired calyx in situations in which the calyx is occupied by a calculus.

To overcome the drawbacks of ultrasound- and fluoroscopy-based techniques for puncture of the renal collecting system, several technologies and techniques have been explored. Each has advantages and disadvantages (Table 1). Bader et al [16] described the use of an optical system incorporated into the percutaneous needle allowing realtime visualization of the renal collecting system, thereby eliminating the need for fluoroscopic guidance. This "allseeing" needle was tested in 15 patients; the puncture had to be repeated in four of them (26\%). The advantage of this system is secure identification of the needle location within the renal collecting system immediately after entry. Visualization of needle entry was aided by ultrasound guidance. However, the system does not allow redirection of the needle in cases of path error. Other disadvantages are difficult visualization in obese patients and a high degree of operator dependence.

An iPad-assisted technique for kidney puncture has been described by Rassweiler et al $[17,18]$. Before the surgical procedure, multislice CT was performed with the patient in the prone position and at the final stage of inspiration. The CT images were analyzed to obtain a 3D reconstruction of the patient anatomy. To choose optimal access to the collecting system, five colored radio-opaque markers were placed around the target area during the CT scan. With the patient under general anesthesia and in the same position in which the preoperative CT was performed, the iPad camera was pointed towards the patient and images of the patient in surgery transmitted by the iPad via Wi-Fi were merged with the virtual preoperative 3D CT images. To allow this image fusion process, at least four markers needed to be visible to the camera. Moreover, since the preoperative CT scan was performed at the final stage of inspiration, the anesthesiologist had to stop the patient breathing at the end of inspiration to perform the puncture in the provided space. Puncture was performed using the virtual 3D image provided and a 2D digital fluoroscopy image in real time to allow final adjustments. This technique was initially tested in a preclinical model using a human phantom, and an error margin of only $1 \mathrm{~mm}$ was recorded. It was then tested clinically in two patients undergoing PCNL. A successful kidney puncture, defined as the needle reaching the desired calyx, was obtained in both cases. This system has the following advantages: correct selection of the puncture location and angle, with good definition of the path; better anatomical knowledge of adjacent organs with 3D images; correct visualization of the patient anatomy regardless of anatomical conformation; minimal space errors; and a shorter time to puncture for training surgeons. Disadvantages include the use of ionizing radiation, the absence of $3 \mathrm{D}$ images in real time, a longer puncture duration for expert surgeons, only minimal adjustments in the path, and the patient in the prone position.

Uro-Dyna-CT (Siemens Healthcare Solutions, Erlangen, Germany) is another technology recently tested for kidney 
Table 1 - Advantages and disadvantages of new techniques for puncture of the renal colleting system

\begin{tabular}{|c|c|c|c|}
\hline Study & Technique & Advantages & Disadvantages \\
\hline Bader et al [16] & $\begin{array}{l}\text { Optical system in the } \\
\text { needle }\end{array}$ & $\begin{array}{l}\text { Secure identification of the needle } \\
\text { Real-time needle visualization via } \\
\text { ultrasound } \\
\text { Easy handling and learning } \\
\text { No ionizing radiation }\end{array}$ & $\begin{array}{l}\text { Needle redirection not possible in the } \\
\text { case of error trajectory } \\
2 \mathrm{D} \text { imaging } \\
\text { Difficult visualization in obese patients }\end{array}$ \\
\hline Rassweiler et al [17] & iPad-assisted puncture & $\begin{array}{l}\text { Correct selection of the needle } \\
\text { location, angle, and trajectory } \\
\text { 3D imaging } \\
\text { Correct visualization of anatomy } \\
\text { Minimal spatial errors } \\
\text { Short learning curve }\end{array}$ & $\begin{array}{l}\text { Use of ionizing radiation } \\
\text { No real-time } 3 \mathrm{D} \text { image } \\
\text { Only minimal adjustments of the path allowed } \\
\text { Patient in prone position }\end{array}$ \\
\hline Ritter et al [19] & $\begin{array}{l}\text { Uro Dyna-CT 3D laser } \\
\text { guidance }\end{array}$ & $\begin{array}{l}\text { 3D anatomy imaging } \\
\text { Quick, safe, and highly accurate } \\
\text { Applicable in complex cases } \\
\text { Trajectory visualization in real } \\
\text { time and possibility of trajectory change }\end{array}$ & $\begin{array}{l}\text { Higher ionizing radiation dose than } \\
\text { conventional technique } \\
\text { Technique hampered by excessive } \\
\text { renal motility } \\
\text { Steep learning curve } \\
\text { High cost }\end{array}$ \\
\hline $\begin{array}{l}\text { Li et al [20] } \\
\text { Chau et al [21] }\end{array}$ & $\begin{array}{l}\text { Ultrasonography using } \\
\text { Sonix GPS navigation } \\
\text { Ultrasonography using } \\
\text { navigation system } \\
\text { under magnetic field }\end{array}$ & $\begin{array}{l}\text { No ionizing radiation } \\
\text { Needle trajectory prediction } \\
\text { Operator can keep track of the needle } \\
\text { during the procedure } \\
\text { Allows adjustment of the trajectory } \\
\text { during the procedure } \\
\text { Easy to perform than conventional } \\
\text { puncture }\end{array}$ & $\begin{array}{l}\text { In obese patients the ultrasound image } \\
\text { is highly impaired, and use of } \\
\text { fluoroscopy is recommended } \\
2 \mathrm{D} \text { image only }\end{array}$ \\
\hline Present study & $\begin{array}{l}\text { Navigation using } \\
\text { electromagnetic sensors }\end{array}$ & $\begin{array}{l}\text { No ionizing radiation } \\
3 \mathrm{D} \text { image of the needle trajectory in } \\
\text { real time } \\
\text { Position and orientation of the needle } \\
\text { in real time } \\
\text { Easy technical learning } \\
\text { Shorter execution time } \\
\text { Ability to redefine the trajectory } \\
\text { Procedure done in supine position. }\end{array}$ & $\begin{array}{l}\text { Lack of visualization of anatomical } \\
\text { structures in the puncture path } \\
\text { Difficult to insert a ueteral catheter } \\
\text { with an electromagnetic sensor in } \\
\text { the desired calyx in situations in } \\
\text { which the calyx is fully occupied } \\
\text { by stones }\end{array}$ \\
\hline
\end{tabular}

puncture $[18,19]$. The Uro-Dyna-CT is a modified angiography unit that allows the fluoroscopic unit to be rotated around the patient, creating a similar image to the images created by CT. Segmentation of the data acquired allows 3D multiplanar reconstruction of the renal collecting system, showing the exact path chosen for puncture. The C-arm is placed in the "bull's eye" position to indicate the puncture site and its direction via a laser light. Once the needle is inserted, the position can be confirmed via conventional fluoroscopy and can be corrected if deemed necessary. Puncture was successful in 9/12 patients tested, and in $7 / 9$ cases the procedure was successful at the first attempt. The average puncture time was $60 \mathrm{~s}$, with an average planning time of $6.5 \mathrm{~min}$ and an average radiation exposure dose of $5850 \mu \mathrm{Gy} / \mathrm{m}^{2}$. This technique provides a 3D anatomical image, and is fast, safe, and highly accurate when there is no patient movement; moreover, the patient can be in the supine position. Disadvantages are higher ionizing radiation doses, a steep learning curve, and high costs. In addition, excessive renal motility hampers the performance.

Ultrasonography using SonixGPS (UltraSonix, Richmond, $\mathrm{BC}$, Canada) navigation is another technological achievement in ultrasound-guided puncture [20]. In this case, a catheter is inserted via cystoscopy in the pyelocaliceal system, with the patient in the lithotomy position. Then, with the patient in the prone position, saline is instilled to distend the pyelocaliceal system. A global positioning system (GPS) electromagnetic transducer is in proximity to the patient. An ultrasound scan is obtained using the SonixGPS ultrasound probe in the area around the 11th and 12 th rib edges, and the images acquired allow identification of a suitable position and direction for the needle during puncture. The direction, depth, and needle insertion angle can be adjusted as needed, according to information available in real time via the needle sensor. Using images obtained via this technique, the needle, which includes an electromagnetic sensor, is guided to the predetermined calyx. This technique was tested in 25 patients with a kidney stone, with $100 \%$ success for puncture at the first attempt and an average puncture time of $5.5 \mathrm{~min}$. It should be noted that none of the patients in this study had a BMI $>35 \mathrm{~kg} / \mathrm{m}^{2}$, as fluoroscopy is still needed in obese patients. Another disadvantage is that the patient anatomy is obtained in 2D. A similar approach was reported by Chau et al [21], who performed 18 PCNL cases using magnetic field-based ultrasound navigation to visualize the position of the needle tract in relation to the target calyx. Puncture was performed freehand without a needle-guiding system attached to the transducer. Needle deviation could be detected and adjusted immediately to achieve a precise puncture. Puncture was successful at the first attempt in $83.3 \%$ of cases.

Comparison of the above techniques to the one described here highlights a few considerations. Puncture techniques 
using electromagnetic sensors and the SonixGPS navigation system had $100 \%$ success at the first attempt [13,19], proving to be the most effective. This high success rate can be explained by the ability of the two systems to define and adjust the needle path in real time. These new techniques allow knowledge of the position of the needle relative to the desired renal calyx for puncture throughout the procedure. The rate of success for puncture at the first attempt was only 73.3\% for the optical system incorporated in the needle [16]; this technique does not provide an ideal path for the needle, which explains the lower rate. In one study of marker-based iPad-assisted puncture, the success rate for puncture at the first attempt was 68.4\% [17]. In this technique it is not possible to redirect the needle during puncture, and the patient must be in the same position during preoperative CT and the procedure, so there may be some errors in the path. The success rate for puncture at the first attempt was $58.3 \%$ using Uro-Dyna-CT for laser-assisted puncture of the renal collecting system [19]. Although this technique shows the direction of the needle trajectory and allows adjustment during the procedure, it is markedly impaired by renal movement caused, for example, by the patient breathing. In an in vitro experiment the same technique had a success rate of $80 \%$ for the first attempt [18], suggesting that it may need to be enhanced before regular use is feasible.

Puncture techniques guided by electromagnetic sensors also seem to offer a shorter learning curve, with only 12 cases required to reach the level of expert surgeon [10], which is much lower than the 60 cases previously reported for standard techniques [6]. Ultrasonography using SonixGPS navigation for puncture of the renal collecting system is also easier than conventional techniques, as the needle position can be monitored throughout the procedure [20]. The optical system incorporated in the needle does not seem to facilitate percutaneous puncture because it only improves visualization when the needle is already in the collecting system. The model that uses an iPad and markers shows that an expert surgeon takes longer to perform a puncture than a training surgeon. This technique is more difficult for an expert surgeon than the conventional approach [17]. The Uro-Dyna-CT technique is also difficult to perform and learn [19].

All the ionizing radiation received during patient diagnosis, treatment, and follow-up is cumulative, and may contribute to a higher risk of malignant transformation [22]. Thus, avoidance of the use of such radiation in PCNL is very important. Puncture of the renal collecting system assisted by electromagnetic sensors effectively avoids ionizing radiation, as the surgeon does not need fluoroscopic guidance. Puncture of the renal collecting system with an optical system incorporated in the needle does not use ionizing radiation because the camera system at the needle tip allows visualization of the renal collecting system in real time, and use of fluoroscopy is not necessary. The SonixGPS system also avoids ionizing radiation by creating the path and by the knowledge of needle positioning in real time. However, the latter two techniques use ultrasound, and in complicated cases (such as obesity and anatomical abnormalities) fluoroscopy must be used because ultra- sound is compromised in these circumstances. The two other techniques rely on ionizing radiation: the average radiation exposure dose is $377.5 \mu \mathrm{Gy} / \mathrm{m}^{2}$ for marker-based iPad-assisted puncture of the renal collecting system [17] and $5850 \mu \mathrm{Gy} / \mathrm{m}^{2}$ for Uro-Dyna-CT for laser-assisted puncture [19]. Both systems have a higher radiation dose than conventional fluoroscopy. Moreover, the Uro-Dyna-CT technique involves a very significant increase in costs [19].

There are a number of limitations of this study that need to be recognized. The sample size is small, as this was conceived as a feasibility study in a highly selected population. Therefore, the technique and technology will need to be tested in more challenging cases, such as large stone burdens filling the renal pelvis and lower pole, as it may be difficult to place the catheter with the electromagnetic sensor in the desired calyx in such cases. In addition, we did not test the system in obese patients. In this regard, we know that the longer the distance between the electromagnetic field generator and the sensor, the more likely is signal loss. Therefore, further investigation in a larger sample and with different study populations is certainly needed.

Moreover, in some patients it might not be possible to introduce the flexible ureterorenoscope because of a tight or narrow ureter. In this scenario we would proceed with a standard technique for kidney puncture, which of course we believe should be part of the surgical skills of anybody performing PCNL.

In addition, one might argue that ureteroscopy-assisted combined access might translate into higher costs in terms of both probes and total resources used. The system itself is not commercially available yet and therefore a specific cost cannot be provided. Moreover, it was beyond the scope of this study to perform a cost assessment, which would require a more complex analysis that should take multiple factors into consideration. Finally, whether there are any harmful effects from these electromagnetic sensors in humans remains undetermined [23].

\section{Conclusions}

A novel navigation system using real-time electromagnetic sensors can be safely and effectively used in the clinical setting for puncture of the renal collecting system during PCNL. This new technology overcomes some of the intrinsic limitations of standard fluoroscopy- and ultrasound-based techniques. These encouraging preliminary findings need to be validated in further clinical investigations using this novel technology.

Author contributions: Estevao Lima had full access to all the data in the study and takes responsibility for the integrity of the data and the accuracy of the data analysis.

Study concept and design: Lima, Rodrigues, João Vilaça.

Acquisition of data: Lima, Mota, Dias, Carvalho.

Analysis and interpretation of data: Lima, Mota, Dias, Carvalho.

Drafting of the manuscript: Lima, Rodrigues.

Critical revision of the manuscript for important intellectual content: Lima, Correia-Pinto, Autorino, João Vilaça.

Statistical analysis: Lima, Autorino. 
Obtaining funding: None.

Administrative, technical, or material support: None. Supervision: Correia-Pinto.

Other: None.

Financial disclosures: Estevao Lima certifies that all conflicts of interest, including specific financial interests and relationships and affiliations relevant to the subject matter or materials discussed in the manuscript (eg, employment/affiliation, grants or funding, consultancies, honoraria, stock ownership or options, expert testimony, royalties, or patents filed, received, or pending), are the following: None.

Funding/Support and role of the sponsor: None.

\section{Appendix A. Supplementary data}

Supplementary data associated with this article can be found, in the online version, at http://dx.doi.org/10.1016/j. eururo.2017.03.011.

\section{References}

[1] Fernstrom I, Johansson B. Percutaneous pyelolithotomy. A new extraction technique. Scand J Urol Nephrol 1976;10:257-9.

[2] Patel SR, Nakada SY. The modern history and evolution of percutaneous nephrolithotomy. J Endourol 2015;29:153-7.

[3] Türk C, Petř́lk A, Sarica K, et al. EAU guidelines on interventional treatment for urolithiasis. Eur Urol 2016;69:475-82.

[4] Assimos D, Krambeck A, Miller NL, et al. Surgical management of stones: American Urological Association/Endourological Society guideline, part I. J Urol 2016;196:1153-60.

[5] Ghani KR, Andonian S, Bultitude M, et al. Percutaneous nephrolithotomy: update, trends, and future directions. Eur Urol 2016;70:382-96.

[6] de la Rosette JJ, Laguna MP, Rassweiler JJ, et al. Training in percutaneous nephrolithotomy. Eur Urol 2008;54:994-1001.

[7] Kalogeropoulou C, Kallidonis P, Liatsikos EN. Imaging in percutaneous nephrolithotomy. J Endourol 2009;23:1571-7.

[8] Seitz C, Desai M, Häcker A, et al. Incidence, prevention, and management of complications following percutaneous nephrolitholapaxy. Eur Urol 2012;61:146-58.

[9] Chen TT, Preminger GM, Lipkin ME. Minimizing radiation exposure during percutaneous nephrolithotomy. Minerva Urol Nefrol 2015;67:347-54.
[10] Rodrigues PL, Vilaça JL, Oliveira C, et al. Collecting system percutaneous access using real-time tracking sensors: first pig model in vivo experience. J Urol 2013;190:1932-7.

[11] Dahm P, Sedrakyan A, McCulloch P. Application of the IDEAL framework to robotic urologic surgery. Eur Urol 2014;65:849-51.

[12] Thomas K, Smith NC, Hegarty N, Glass JM. The Guy's stone scoregrading the complexity of percutaneous nephrolithotomy procedures. Urology 2011;78:277-81.

[13] Scoffone CM, Cracco CM, Cossu M, et al. Endoscopic combined intrarenal surgery in Galdakao-modified supine Valdivia position: a new standard for percutaneous nephrolithotomy? Eur Urol 2008;54:1393-403.

[14] Grasso M, Lang G, Taylor FC. Flexible ureteroscopically assisted percutaneous renal access. Tech Urol 1995;1:39-43.

[15] Alsyouf M, Arenas JL, Smith JC, et al. Direct endoscopic visualization combined with ultrasound guided access during percutaneous neohrolithotomy: a feasibility study and comparison to a conventional cohort. J Urol 2016;196:227-33.

[16] Bader MJ, Gratzke C, Seitz M, et al. The "all-seeing needle": optical puncture system confirming access in percutaneous nephrolithotomy. Eur Urol 2011;59:1054-9.

[17] Rassweiler JJ, Müller M, Fangerau M, et al. iPad-assisted percutaneous access to the kidney using marker-based navigation: initial clinical experience. Eur Urol 2012;61:628-31.

[18] Rassweiler J, Rassweiler MC, Müller M, et al. Surgical navigation in urology: European perspective. Curr Opin Urol 2014;24:81-97.

[19] Ritter M, Rassweiler MC, Michel MS. The Uro Dyna-CT enables three-dimensional planned laser-guided complex punctures. Eur Urol 2015;68:880-4.

[20] Li R, Li T, Qian X, et al. Real-time ultrasonography-guided percutaneous nephrolithotomy using SonixGPS navigation: clinical experience and practice in a single center in China. J Endourol 2015;29:158-61.

[21] Chau HL, Chan HC, Li TB, Cheung MH, Lam KM, So HS. An innovative free-hand puncture technique to reduce radiation in percutaneous nephrolithotomy using ultrasound with navigation system under magnetic field: a single-center experience in Hong Kong. J Endourol 2016;30:160-4.

[22] Mahesh M. Fluoroscopy: patient radiation exposure issues. Radiographics 2001;21:1033.

[23] Kovacic P, Somanathan R. Electromagnetic fields: mechanism, cell signaling, other bioprocesses, toxicity, radicals, antioxidants and beneficial effects. J Recept Signal Transduct Res 2010;30:214-26. 